\title{
Technology for Successful Aging
}

\author{
Harry W. Tyrer ${ }^{1}$ PhD, Member IEEE, Majd Alwan PhD, Senior Member IEEE, George Demiris, Zhihai (Henry) He, \\ Senior Member IEEE, Jim Keller, Fellow IEEE, Marjorie Skubic PhD, Member IEEE, Marilyn Rantz PhD
}

\begin{abstract}
Americans are living longer, and research shows that seniors are embracing independence, and will benefit from living in the same place. These are the rationale for 'Aging in Place' and the development of Tiger Place, an "Aging in Place ' Environment in Columbia Missouri. Our goal is to minimize intrusion, allow the resident complete control over privacy and treatment (if any), and to provide substantive improvement in quality of life. Nevertheless there continues to be significant risks to the elderly which results in reduced functional and cognitive activity. While there has been much technology developed to ameliorate these factors, there is no comprehensive evaluation of the benefit of these devices nor a comprehensive strategy to improve the quality of life of seniors as determined by functional ability and possibly later cognitive ability.

With our partners at the University of Virginia we are developing a system of sensors, to monitor the activity of seniors in their residences. We measure motion, footfalls, sleep and restlessness, we have stove sensors and sensing mats, all connected wirelessly to a computer which performs an initial evaluation and data transfer to a secure server for further study. Based upon the monitor data we will implement an intervention to ameliorate functional decline. Focus group studies determine the attitudes, concerns and impressions of the residents and staff. We find that senior's attitude to technology is healthy and they will try helpful approaches. In addition to the statistical comparisons, we model the data using hidden Markov models, integrate or fuse the monitor data with
\end{abstract}

\footnotetext{
${ }^{1}$ Manuscript received April 3, 2006, The authors are grateful for the support from NSF ITR grant IIS-0428420 and the U.S. Administration on Aging, under grant 90AM3013.
}

Harry W. Tyrer is professor in Electrical and Computer Engineering at the University of Missouri - Columbia He is the corresponding author Please use email tyrerh@missouri.edu, Day phone is 573882 - 6489, shipping address is 305 Engineering Building West, University of Missouri - Columbia; Columbia, Missouri 65211

Majd Alwan is with the Medical Automation Research Center (MARC) at the University of Virginia - Charlottesville

George Demiris is Assistant Professor in Health Management and Informatics at the school of Medicine at The University of Missouri Columbia

Zhihai (Henry) He professor in Electrical and Computer Engineering at the University of Missouri - Columbia

Jin Keller professor in Electrical and Computer Engineering at the University of Missouri - Columbia

Marjorie Skubic is Associate Professor in Electrical and Computer Engineering at the University of Missouri - Columbia

Marilyn Rantz is Professor, Sinclair School of Nursing, University of Missouri-Columbia video images, and reason about behavior using fuzzy logic. The results of this work will additionally reduce the workload on caregivers, foster communication between residents and family, and give these seniors independence. We have requested and received IRB approval for this study.

\section{INTRODUCTION}

The predominant paradigm in long-term care services for older adults is relocation to another setting as increased care becomes necessary [1]. Relocation is not a benign event in the lives of older adults, $90 \%$ of whom have at least one chronic health condition. Relocation may precipitate acute illness, exacerbate chronic illness, contribute to anxiety and depression, and adversely impact perceived quality of life [2]. Further. older adults and their families resist relocation to nursing homes. Nursing homes are perceived as unpleasant, restrictive institutional environments that are not homelike and that neither respect nor encourage the individuality of the residents [3,4]. In 1996, influenced by the consumer perspective, MU Sinclair School of Nursing (SSON) faculty began developing a new model of long-term care for older adults that resulted in the Aging in Place Project. Aging in Place Project allows seniors to age in place in the home environment of their choice with supportive health care services as needed [5-8]

TigerPlace implements aging in place and is a unique eldercare setting near the campus of The University of Missouri-Columbia (MU). Americare Systems, Inc., of Sikeston, Missouri manages TigerPlace a 34,000 square foot facility with 32 apartment units and occupation which began in April 2004 [9]. TigerPlace makes possible for multidisciplinary teams of nurses, engineers and informatics specialists to develop and study the technological interventions described in this proposal [9].

Due to its clinical focus, the TigerPlace project encompasses a broader approach to individualized technology than that of the "smart home" concept which is an emerging trend in health informatics. Smart home features usually include motion-sensing devices for automatic lighting control, motorized locks, door and window openers, motorized blinds and curtains[10], smoke and gas detectors and temperature control devices. Such an infrastructure is designed to address some of the neurological and cognitive problems in the elderly, and enhance their ability to function independently within their residence. Smart home initiatives include the SmartBo 
project[10], a demonstration apartment operated by the Swedish Handicap Institute; the PROSAFE project ${ }^{13}$ that utilizes a set of infrared motion sensors connected to either a wireless or wired network to support clinical nursing assessment for risks of functional decline and alerting mechanisms of the need for intervention promoting as much independence and autonomy as possible.

\section{MODEL OF FUNCTIONAL AND COGNITIVE DECLINE}

Older adults decline in mobility, cognition and the senses at varying rates. An important goal in the care of the elderly is to help them remain independent, to do so we must minimize their decline. In our conceptual model (Fig. 1), mobility and cognitive impairments lead to functional decline. Sensory changes -- especially changes in vision, hearing, and balance-- mediate the nature and extent of mobility and cognitive impairments and the severity of functional decline. Mobility is essential for independence in activities of daily living: being able to get out of a bed or chair, move to the bathroom, reach food and fluids, and reach a telephone are essential tasks that require functional ability.

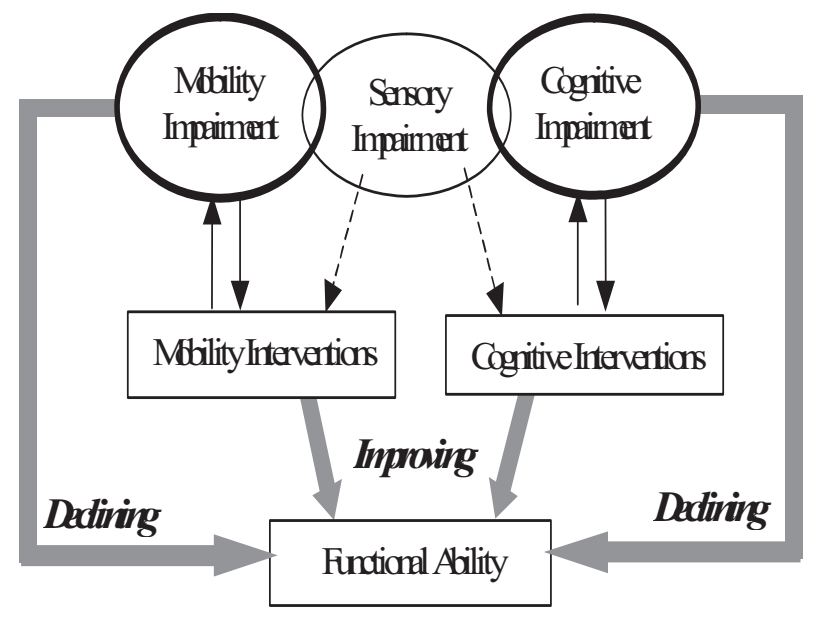

Fig. 1. Functional decline and functional improvement from interventions

Mobility issues such as impaired gait and balance limit physical activity and contribute to functional decline [11]. When older adults ranked the five most important skills as balance, ability to see, ability to lock and unlock doors, using a toilet, and managing medications. As functional independence in mobility declines, older adults are at increased risk of falls, a major cause of disability and death. A downward spiral begins with a fall and subsequent loss of confidence. Falls are the $7^{\text {th }}$ leading cause of death for older adults. Over $50 \%$ of falls lead to minor injuries while approximately $2 \%$ of falls lead to hip fractures and approximately $5 \%$ of falls lead to other fractures [11-13].

The cognitive faculties that prompt and guide activities are equally important in preserving independence. Cognitive impairments include decreases in recall of instructions, ability to follow complex instructions and problem solving ability. Older adults with impaired cognition, such as those with Alzheimer's disease and even mild dementia, may forget operating instructions for activities, including how to ask for assistance and recognize unsafe situations, difficulty self-administration of medications, and even telephone use may become difficult. As cognitive impairment increases over time, as in dementia, dependence in activities of daily living and inability to be left alone safely may lead to institutionalization.

\section{TeChNOlogy AND Tiger Place}

The low cost of technology and its ubiquity provides an enabling resource to mitigate risk to seniors and to ameliorate their loss of function as they age in place.

\section{A. Sensor Systems}

MARC's In-Home Monitoring System is comprised of motion sensors in every room, including the bathroom, and a motion sensor dedicated to the shower area, a stove-top temperature sensor, and a bed sensor system that all transmit data wirelessly to a Personal Computer based Data Manager. The bed sensor detects presence, pulse, and movement in bed. Pulse is computed from a bed pad signal while the monitored individual is quiescent in bed; movement artifacts prevent pulse measurements, but provide information on restlessness. A passive floor-vibration based gait monitor and fall detector produces signals that are processed to detect the falss or gait. Moreover, each system is enhanced with the ability to automatically notify caregivers upon the detection of conditions consistent with possible emergency situations $[14,15]$.

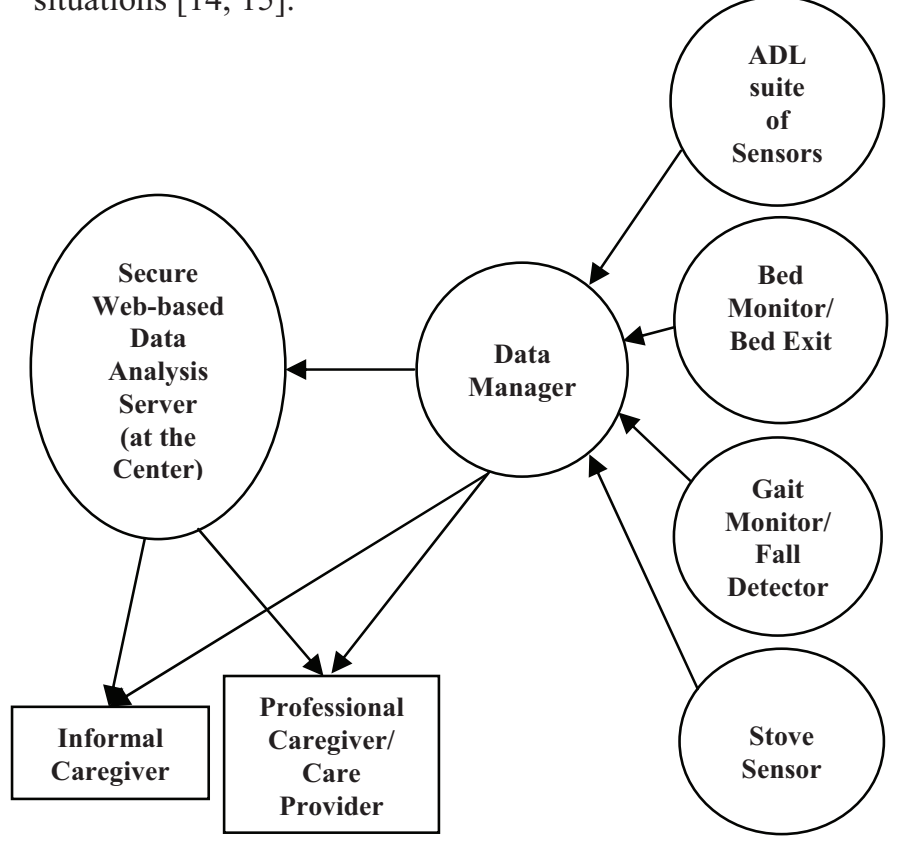

Fig. 2. The Common Modular Architecture of MARC's In-Home Monitoring System

The Data Manager collects data from separate sensor modules; processes alert conditions locally, time-stamps and 
logs the collected data. Upo0n meeting the pre-determined alert conditions, the Data Manager can immediately page the caregiver. Alerts are also registered into the data log. Data are automatically processed by the activity inference software that detects key ADLs including meal preparation, showering, and bathroom visits. Professional caregivers of monitored older adults can access summary reports listing all the participating residents under their care with a priority score reflecting each monitored individual's potential need for attention.

The Data Manager monitors four alert conditions that include "possible forgotten stove burner", high or low pulse, "possible fall", as well as an "impact fall" alert condition, indicated by a signal from a fall detector. Such a notification sub-system, unlike many emergency pendants, does not require user activation. The two-stage possible fall notification sub-system is based on lack of activity; it monitors motion sensors in every room, as well as bed exit from the bed sensor. A possible fall "watch" starts whenever the resident exits the bed, and a "possible fall" alert is reported if lack of motion persists for a pre-determined period following bed exit. The fall watch remains active until the participant leaves the bedroom and bathroom area (indicated by reported motion activity outside the bathroom or bedroom) or returns to bed (indicated by movement followed by detection of the pulse). With no motion detected after exiting the bed, the Data Manager can connect to the facility's pager system and sent the participant's identification code appended with the code for a "possible fall" to the caregiver [17]. Fig.2. illustrates the system's architecture.

\section{1) Previous studies}

MARC's In-Home Monitoring System, together with its activity inference algorithms, was initially tested for 18 months under an institutional review board (IRB) approved study in a private home that served as our "living laboratory". In these preliminary studies, the activity data of a normal healthy middle-aged participant was logged and analyzed using several data analysis techniques, including clustering, mixture models [14] and a rule-based approach, where spatial-temporal relationships among sensor events are exploited to infer the occurrence of activities with a high degree of confidence. The latter approach was adopted for the inference of the activities of interest. The system's rulebased inference methods were validated against 37 days of the subject's self-report, recorded in real-time using a Personal Digital Assistant (PDA) based electronic diary developed specifically for the validation study. A detailed description of the activity inference rules and validation results are reported in [15]. The knowledge-acquired rules were also compared to automated supervised learning methods including, decision trees and Random Forests, and were found to be more reliable [16]. The acceptance, utility, and impact of the system on the different stake-holders including older adults, professional caregivers and informal caregivers were evaluated in a series of pre-experimental pilot studies in different care settings and presented in [17$20]$.

An extension of the pilot reported in [17] revealed that professional caregivers were able to utilize the monitoring data preventively. For example, at the earliest signs of increased restlessness coupled with more frequent nightly bathroom visits, an indicator of a potential urinary tract infection, caregivers intervened by increasing that resident's fluid intake, which can clear up the infection without pharmaceutical or clinical intervention.

\section{B. Residents' Perceptions of the TigerPlace technology}

One of the objectives of this project is to evaluate the usability of the technology and investigate fundamental issues in human-computer interaction for the population of older adults. The evaluation plan consists of observations, focus groups and interviews with representative users in the target group of older adults in TigerPlace.

\section{1) Observations}

We are conducting a series of heuristic observations onsite to identify problems and usability issues of the technology. Heuristic evaluation, is a usability inspection method, which refers to a class of techniques in which evaluators examine an interface for usability issues. Observation is considered an informal method of evaluating usability because observers rely on heuristics along with their experience and the knowledge.

2) Focus groups

In order to gain insight into users' perceptions of the technology, we are conducting a limited number of focus groups. We have developed a protocol to examine users' perceptions and satisfaction. Topics for discussion include: satisfaction with the intervention, barriers and facilitators to use of the technology, confidence in using this form of intervention. Focus groups take place at regular intervals from the beginning of the study until completion. This repetition allows us to assess the diachronic reliability of the focus group protocol (as a test-retest reliability index). Each focus group session has approximately 4 to 6 participants and lasts about one hour. We tape-record sessions, transcribe the tapes and use QSR N6 software to manage and interpret the data. The software helps organize emerging ideas, search and explore in context, seek patterns and meanings, construct and test explanations and relate theories to data.

\section{3) Interviews}

We are developing a survey instrument to assess users' satisfaction with the technology infrastructure and instrumentation components. Although this will render a score for the level of satisfaction, we will focus on assessing users' perceptions. The instrument will be a structured survey to be used during a structured interview or to be filled out by each user. Responses will indicate the level of agreement on a Likert scale. The Instrument addresses the following 
- The resident's operation of technology including ease of use, adjusting to changing needs, and maintenance,

- impact on lifestyle, including restraints on mobility and interference with other activities,

- psychological factors, including confidence in using the intervention, reliability of the system, trust in its accuracy and performance, sense of control and concern for violation of privacy.

When applicable, the instrument will address the impact of the intervention on caregivers and family members. The items will refer to all areas of application, namely the monitoring system with motion sensors, sensor mats and gait monitors; event-driven, anonymized video sensor; and recognition of behavioral patterns. Specifically for the alert display system and the system for generating behavior rules, emphasis will be placed on assessing users' perception of its friendliness, ease of following instructions and willingness to utilize this feature on a daily basis.

4) Preliminary Results

We have conducted three focus group sessions with a total of 12 participants and two observation sessions. Preliminary findings indicate that residents have a very positive attitude towards smart home technologies in general, and the sensor-based interventions of TigerPlace more specifically. Residents perceive the technology as nonintrusive, and not interfering with daily activities. Privacy concerns were only expressed in relation to the video-sensor network by 3 participants. The two observation sessions allow for validity testing but also an assessment of the level of interference of the technology with daily activities. The two observation sessions included specific activities (meal preparation); two observers recorded all tasks and timed them. The observation findings were then compared to the sensor-generated data to ensure that the sensors fired signals at the appropriate intervals and that the collection of data can indicate the type of activity (in this case, meal preparation).

\section{REFERENCES}

[1] (Frank 2001). How long can I stay?: The dilemma of aging in place in assisted living. In B. Schwarz (Ed.), Assisted living: Sobering realities. New York: Haworth Press.

[2] Manion 1995) \& Rantz, M.J. 1995. Relocation stress syndrome: A comprehensive plan for long term care admissions. Geriatric Nursing, 16(3), 108-112.

[3] (Rantz 1998) Mehr, D., Popejoy, L., Zwygart-Stauffacher, M., Hicks, L., Grando, V., Conn, V., Porter, R., Scott, J., \& Maas, M. (1998). Nursing home care quality: A multidimensional theoretical model. Journal of Nursing Care Quality, 12(3), 30-46. *

[4] (Rantz 1999) Zwygart-Stauffacher, M., Popejoy, L., Grando, V., Mehr, D., Hicks, L., Conn, V., Wipke-Tevis, D., Porter, R., Bostick, J. \& Maas, M. 1999. Nursing home care quality: A multidimensional theoretical model integrating the views of consumers and providers. Journal of Nursing Care Quality, 14(1), 16-37*

[5] (Marek 2000) Rantz, M.J. 2000. Aging in Place: A New Model for Long Term Care. Nursing Administration Quarterly, 24(3), 1-11.

[6] Marek, (2004), Rantz, M.J., \& Porter, R. 2004. Senior Care: Making a difference in long-term care of older adults. Journal of Nursing Education, 43(2): 81-83.
[7] (Rantz 2000) Marek, K.D., \& Zwygart-Stauffacher, M. 2000. The future of long-term care for the chroniacally ill. Nursing Administration Quarterly, 25(1), 51-58.

[8] (Rantz 2003). Aging in place. Nurseweek Midwest/Heartland Edition, $4(2), 7$.

[9] (Rantz 2004b) Rantz, M.J., Marek, K.D., Aud, M. A., Tyrer, H. W., Skubic, M., Demiris, G., \& Jussam, A.A. (in press). Technology and nursing collaboration to help older adults age in place. Nursing Outlook. In press.

[10] (Elger 1998), Furugren B. SmartBo-an ICT and computer-based demonstration home for disabled people. Proceedings of the $3^{\text {rd }}$ TIDE Congress: Technology for Inclusive Design and Equality Improving the Quality of Life for the European Citizen. Helsinki, Finland June 1998.

[11] Myers, A. H., Young, Y., \& Langlois, J. A. 2002. "Prevention of falls in the elderly," Bone, 18, 87S-101S.

[12] Friedman, S. M., Munoz, B., West, K. K., Rubin, G. S., \& Fried, L. P. (2002). Falls and fear of falling: Which comes first? A longitudinal prediction model suggests strategies for primary and secondary preventions. Journal of the American Geriatrics Society, 50, 13291335.

[13] Tideiksaar R. 2002. Falls in older people: Prevention and management (3rd ed.). Baltimore: Health Professions Press.

[14] T Barger, D Brown, M Alwan. Health Status Monitoring Through Analysis of Behavioral Patterns. IEEE Trans. on Systems, Man and Cybernetics (Part A: Systems and Humans), 2005; Vol. 35, No. 1, pp 22-27.

[15] M Alwan, J Leachtenauer, S Dalal, et al. Validation of Rule-Based Inference of Selected Independent ADLs. Accepted for publication in the Journal of Telemedicine and E-Health, Oct. 2005, Vol. 11, No. 5: 594-599.

[16] S Dalal, M Alwan, R Seirafi, S Kell, D Brown. A Rule-Based Approach to Analyzing Elders' Activity Data: Detection of Health and Emergency Conditions, Proceedings of the AAAI's Fall Symposium on AI \& Eldercare: Caring Machines, Washington, DC, November 2005.

[17] M Alwan, S Dalal, D Mack, S Kell, B Turner, J Leachtenauer, R Felder. Impact of Monitoring Technology in Assisted Living: Outcome Pilot. Accepted for publication in the IEEE Transaction on Information Technology in BioMedicine, Vol. 10, No. 1, January 2006, pp. 192-198.

[18] M Alwan, D Mack, S Dalal, S Kell, b Turne, R Felder. Impact of Passive In-Home Health Status Monitoring Technology in Home Health: Outcome Pilot, In Proceedings of the Transdisciplinary Conference on Distributed Diagnosis and Home Healthcare (D2H2), 2 - 4 April 2006, Arlington, VA.

[19] M Alwan, J Leachtenauer, S Dalal, D Mack, S Kell, B Turner, R Felder. Psychosocial Impact of Passive Health Status Monitoring Technology in Assisted Living: A Pilot Study, To Appear in Proceedings of the 2nd IEEE International Conference on Information \& Communication Technologies: From Theory to Applications (ICTTA'06), April 23rd-28th 2006, Damascus, Syria.

[20] M Alwan, S Kell, B Turner, S Dalal, D Mack, Felder R. Psychosocial Impact of Passive Health Status Monitoring on Informal Caregivers and Older Adults Living in Independent Senior Housing, To Appear in Proceedings of the 2nd IEEE International Conference on Information \& Communication Technologies: From Theory to Applications (ICTTA'06), April 23rd-28th 2006, Damascus, Syria

[21] M. Alwan, S. Dalal, S. Kell, R. Felder. Derivation of Basic Human Gait Characteristics from Floor Vibrations. 2003 Summer Bioengineering Conference, June 25-29, Sonesta Beach Resort in Key Biscayne, Florida.

[22] P. Rajendran, M. Alwan, S. Kell, et al. A Passive Floor-Vibration Based Fall Detector, Accepted in the International Conference on Aging, Disability and Independence, to be held in Florida Feb. 2006.. 